\title{
TOPOLOGICAL LOOPS WITH INVARIANT UNIFORMITIES
}

\author{
BY \\ SIGMUND HUDSON(1)
}

1. Introduction. A topological loop $\mathscr{L}$ is said to have a right (left) invariant uniformity $\mathscr{U}$ if there exists a uniform structure $\mathscr{U}$ compatible with the topology of $\mathscr{L}$ such that for any $a \in \mathscr{L}$ and any entourage $A \in \mathscr{U},(x, y)$ belongs to $A$ if and only if $(x a, y a)$ (respectively, $(a x, a y))$ belongs to $A$. In the case that $\mathscr{L}$ is a group, this terminology is consistent with that of topological groups. K. H. Hofmann [6] has shown that a compact topological loop has a uniform structure $\mathscr{U}$ having the property that for any $a \in \mathscr{L}$ and any entourage $A \in \mathscr{U}$, there exists an entourage $B \in \mathscr{U}$ contained in $A$ such that $(x, y) \in B$ implies that $(a x, a y),(x a, y a)$, $\left(\left(a^{-1} x\right),\left(a^{-1} y\right)\right)$, and $\left(\left(x a^{-1}\right),\left(y a^{-1}\right)\right)$ all belong to $A\left(^{2}\right)$. There exist loops whose topologies are that of a Euclidean 1-sphere which do not have a left or a right invariant uniform structure (see $\S 5$ ). As in the case of topological groups, a loop with a right invariant uniformity which is metrizable has a right invariant metric.

The purpose of this paper is, firstly, to make use of a "natural tool," namely, a "group of translates," for the study of certain loops possessing various uniformities. It is shown in Theorem 1 that:

A compact loop $\mathscr{L}$ has an invariant uniformity if and only if the group of all translates of $\mathscr{L}$ is relatively compact.

Next, with the aid of the group of translates it is proved in Theorem 4 that:

$A$ loop with an invariant uniformity on a Euclidean $n$-sphere is isomorphic to one of the following: the cyclic group of order two, or the complex, the quaternion, or the Cayley numbers of norm one, respectively.

Finally, using this result on $n$-spheres with invariant uniformity, it is shown that certain diassociative loops with zero on Euclidean $n$-spaces are isomorphic to the multiplicative structure of either the real, complex, quaternion, or Cayley numbers

The author is grateful for the many suggestions and kindness of Professor Paul Mostert and Dr. Karl Heinrich Hofmann.

2. Invariant uniformities. In the study of a topological loop $\mathscr{L}\left({ }^{3}\right)$, the

Received by the editors September 17, 1962.

(1) The author is grateful to the National Science Foundation for partial support given via grants to faculty members of Tulane University. This work will be submitted as a portion of the author's doctoral dissertation under the direction of Professor Paul S. Mostert.

(2) In a loop, $\left(a^{-1} b\right)$ denotes the solution for $x$ of the equation $a x=b$ for given $a$ and $b$, and $\left(b a^{-1)}\right.$ the solution for $y$ of the equation $y a=b$.

(3) See [6], for example, for definition. 
right (left) translate by $a \in \mathscr{L}$ is the homeomorphism of $\mathscr{L}$ onto itself, denoted by $R_{a}$ (respectively, $L_{a}$ ), that maps $x \in \mathscr{L}$ onto $x a$ (respectively, ax). The set of all right translates generates a subgroup of the group of all homeomorphisms of $\mathscr{L}$ onto itself. Similarly the set of all left translates and the set of all translates (both right and left) generate subgroups. If $\mathscr{L}$ is locally compact, then the three abovementioned groups are transitive, topological transformation groups of $\mathscr{L}$ when given the $g$-topology [1].

THEOREM 1. Let $\mathscr{L}$ be a compact loop and $\mathfrak{5}$ denote the group generated by all right and left translates of $\mathscr{L}$. Then $\overline{\mathfrak{5}}$, the closure of $(\mathfrak{5}$ in the set of all continuous functions from $\mathscr{L}$ to $\mathscr{L}$ with the g-topology (equivalently, in this case, the compact-open topology), is a compact, transitive transformation group of $\mathscr{L}$ if and only if $\mathscr{L}$ has an invariant uniformity.

Proof. First suppose that $\mathscr{L}$ has an invariant uniformity $\mathscr{U}$. Ascoli's theorem will be applied to show $\overline{\mathscr{F}}$ is compact. It is sufficient to show that $\mathfrak{G}$ is equicontinuous and, secondly, $x \in \mathscr{L}$ implies that $\mathfrak{5}(x)$, the orbit at $x$, is relatively compact in $\mathscr{L}$. The second condition obviously holds, as does the transitivity of $\overline{\mathfrak{G}}$. To show the first condition holds, if $a \in \mathscr{L}, f \in \mathfrak{F}$, and $A$ is any entourage of $\mathscr{U}$, then

$$
f=R_{a_{1}} L_{a_{2}} R_{a_{3}}^{-1} L_{a_{4}}^{-1} \cdots R_{a_{n-3}} L_{a_{n-2}} R_{a_{n-1}}^{-1} L_{a_{n}}^{-1}
$$

for some $a_{1}, \ldots, a_{n} \in \mathscr{L}$. Using the invariance property of the uniformity of $\mathscr{L}$ and inducting on $n$, it is easily seen that $(a, x) \in A$ implies that $(f(a), f(x)) \in A$. Hence $f(A(a)) \subset A(f(a))$ and $\mathfrak{b}$ is equicontinuous. It is noted that to show $\mathfrak{F}$ equicontinuous it would be sufficient to assume the weaker property that the uniformity of $\mathscr{L}$ satisfy the property that for any entourage $A$ there exists an entourage $B \subset A$ such that $(x, y) \in B$ implies $(f(x), f(y)) \in A$ for all $f \in \mathbb{F}$.

Furthermore $\overline{\mathfrak{G}}$ is a group; for let $g \in \overline{\mathfrak{G}}$. There is a net $\left\{g_{\alpha}\right\}$ converging to $g$ with each $g_{\alpha}$ belonging to $\mathfrak{F}$. Since $\overline{\mathfrak{F}}$ is compact, $\left\{g_{\alpha}^{-1}\right\}$ has a convergent subnet $\left\{g_{\alpha_{\beta}}^{-1}\right\}$. Hence $g$ has a right and left inverse in $\overline{\mathfrak{G}}$, namely, $\lim _{\beta} g_{\alpha_{\beta}}^{-1} \cdot \overline{\mathfrak{G}}$ is algebraically closed under multiplication. It is easily seen that $\overline{\mathfrak{G}}$ is a topological semigroup, and it follows that $\overline{\mathbb{F}}$ is a topological group since $\overline{\mathbb{5}}$ is compact [4].

Conversely, suppose that $\overline{\mathscr{G}}$ is a compact transformation group of $\mathscr{L}$. Then $\overline{\mathbb{G}}$ has an invariant uniformity. The right coset space $\overline{\mathfrak{G}} / \overline{\mathfrak{F}}_{1}$ (for 1 , the identity of $\mathscr{L}$, $\overline{\mathfrak{F}}_{1}$ is the subgroup of all $f \in \overline{\mathfrak{F}}$ for which $f(1)=1$ ) has a right invariant uniformity, the one induced from $\overline{\mathfrak{G}}$. Since $\overline{\mathfrak{G}}$ is compact, the function from $\overline{\mathscr{G}} / \overline{\mathfrak{G}}_{1}$ onto $\mathscr{L}$ mapping $\overline{\mathfrak{G}}_{1} g$ onto $g(1)$ is a homeomorphism; and it follows that $\mathscr{L}$ has a right invariant uniformity. Similarly the left coset space has a left invariant uniformity and $\mathscr{L}$ has also left invariant uniformity. Since $\mathscr{L}$ is compact, these two uniformities are the same.

The following corollary follows from the above remarks.

COROLlaRY. If $\mathscr{L}$ is a compact loop, then $\mathscr{L}$ has an invariant uniformity if 
and only iffor any entourage $A$ there exists an entourage $B \subset A$ such that $(x, y) \in B$ implies $(f(x), f(y)) \in A$ for all $f \in \mathfrak{G}$.

The notation introduced in Theorem 1 will be observed throughout this note. In particular, $\overline{\mathfrak{F}}$, rather than $\mathfrak{G}$, will be called the group of all translates of $\mathscr{L}$.

THEOREM 2. Let $\mathscr{L}$ be a locally compact, connected loop. Then $\mathbb{( 5}$ and $\overline{\mathfrak{G}}$ are connected spaces.

Proof. Define a function $F: \mathscr{L} \rightarrow \mathfrak{G}$ by $F(x)=R_{x}$. If $\left\{x_{\alpha}\right\}$ is a net in $\mathscr{L}$ converging to $x \in \mathscr{L}$, then $R_{x_{\alpha}}\left(y_{\beta}\right)=y_{\beta} x_{\alpha}$ converges to $y x$ whenever $\left\{y_{\beta}\right\}$ converges to $y$, because of continuity of multiplication in $\mathscr{L} . R_{x_{x}}^{-1}$ also converges to $R_{x}^{-1}$. Hence $F\left(x_{a}\right)$ converges to $F(x)=R_{x}$ in the $g$-topology [1], and $F$ is continuous. Similarly the function from $\mathscr{L}$ to $\mathfrak{G}$ mapping $x$ onto $L_{x}$ is continuous. For each natural number $n$, define a function $T_{4 n}$ from $\mathscr{L}^{4 n}$ (the $4 n$-fold cartesian product of $\mathscr{L}$ by itself) to $(5$ by

$$
T_{4 n}\left(x_{1}, \cdots, x_{4 n}\right)=R_{x_{1}} L_{x_{2}} R_{x_{3}}^{-1} L_{x_{4}}^{-1} \cdots R_{x_{4 n-1}}^{-1} L_{x_{4 n}}^{-1} .
$$

From the previous remarks on the continuity of $F$ and the fact that multiplication and inversion are continuous in $\mathfrak{G}$, it follows that $T_{4 n}$ is continuous. Hence $T_{4 n}\left(\mathscr{L}^{4 n}\right)$ is connected for each $n$. Since $\left(\mathfrak{H}=\bigcup\left\{T_{4 n}\left(\mathscr{L}^{4 n}\right): n=1,2, \ldots\right\}\right.$ and the identity of $\mathfrak{G}$ belongs to each $T_{4 n}\left(\mathscr{L}^{4 n}\right), \mathfrak{G}$ is connected; and $\overline{\mathfrak{F}}$ is also connected.

The proof of Theorem 2 implies that, if $\mathscr{L}$ is compact, then $\mathfrak{G}$ is the countable union of a tower of compact sets.

THEOREM 3. If $\mathscr{L}$ is a locally compact, connected loop with an invariant uniformity, then $\overline{\mathbb{5}}$ is a locally compact transformation group.

The proof of this theorem follows from [1, Theorem 7].

The above statements may be appropriately modified for right (left) invariant uniformities and the group generated by the right (respectively, left) translates. Theorems 1 and 2 have interest in the case that $\mathscr{L}$ is additionally a nonabelian group.

3. Loops on $n$-spheres. In the section the results of the second section are applied to loops on $n$-spheres with an invariant uniformity.

THEOREM 4. Let $\left(\mathscr{L},{ }^{\circ}\right)$ be a loop with an invariant uniformity whose topology is that of a Euclidean $n$-sphere $S^{n}$. Then $(\mathscr{L}, \circ)$ is isomorphic to one of the following: the cyclic group of order two, the complex numbers of norm one, the quaternions of norm one, or the Cayley numbers of norm one.

Proof. We assume $n>0$, for a two element loop is a group. We may also assume that $S^{n}$ is the unit sphere in $R^{n+1}$, Euclidean $(n+1)$-dimensional space. The purpose of the following constructions is to obtain a division algebra over the reals, where the multiplication in the division algebra is obtained from that of $\left(\mathscr{L},{ }^{\circ}\right)$. 
Let $\overline{\mathscr{G}}$ be the group of translates of $\mathscr{L}$. Then $\overline{\mathscr{G}}$ is a compact, connected, effective, transitive transformation group of homeomorphisms acting on the $n$-sphere. Hence $\overline{\mathbb{F}}$ is a Lie group [9]. For any $g \in \overline{\mathbb{F}}$ we extend the domain of the mapping $g$ to $R^{n+1}$ by defining $g(O)=O$ and $g(x)=a g\left(x^{\prime}\right)$, where $O \neq x \in R^{n+1}, x=a x^{\prime}, a$ is a positive real number, and $x^{\prime}$ is the unique vector on the $n$-sphere lying on the ray starting at the origin through $x$ (where $a g\left(x^{\prime}\right)$ is the usual Euclidean scalar product of $a$ by the vector $\left.g\left(x^{\prime}\right)\right)$. Hence the elements of $\overline{\mathfrak{F}}$ are transformations of $R^{n+1}$ and it is easily verified that $\overline{\mathfrak{G}}$ is a topological transformation group of $R^{n+1}$.

$\overline{\mathfrak{G}}$ is a compact, connected Lie group acting effectively on $R^{n+1}$ and has at least one $n$-dimensional orbit. By a theorem of J. Poncet [11] there exists a homeomorphism $\pi$ of $R^{n+1}$ into $E^{n+1}$ (also Euclidean $(n+1)$-space) and an isomorphism $H$ of $\overline{\mathbb{5}}$ into $\mathrm{SO}(n+1)$ such that for any $g \in \overline{\mathbb{G}}$ and any $x \in R^{n+1}$

$$
\pi(g(x))=[H(g)](\pi(x)),
$$

where the element $H(g)$ of $\mathrm{SO}(n+1)$ acts on $\pi(x)$ in $E^{n+1}$ in the usual manner of matrix multiplication.

It follows from equation (1) and from the fact that $\pi$ is a homeomorphism, that the origin is mapped upon the origin under $\pi$ and orbit spheres about the origin are mapped onto such. We may thus assume that the unit sphere in $R^{n+1}$ is mapped on the unit sphere in $E^{n+1}$, because we may follow $\pi$ with a linear transformation which preserves equivalence of transformation groups. Define a multiplication on the unit sphere $\pi(\mathscr{L})$ in $E^{n+1}$ by

$$
x \cdot y=\pi\left(\pi^{-1}(x) \circ \pi^{-1}(y)\right) .
$$

It is obvious that $\mathscr{L}$ and $\pi(\mathscr{L})$ are isomorphic as topological loops. We extend the multiplication of $\pi(\mathscr{L})$ to $E^{n+1}$ in the following manner. If $x, y$ are vectors in $E^{n+1}$, both nonzero, $x$ and $y$ may be represented uniquely as $x=a x^{\prime}, y=b y^{\prime}$; where $a$ and $b$ are real numbers, $x^{\prime}$ and $y^{\prime}$ are on the unit $n$-sphere, $x^{\prime}$ is on the ray starting at the origin through $x$, and $y^{\prime}$ is on the ray starting at the origin through $y$. Define $x \cdot y=a b\left(x^{\prime} \cdot y^{\prime}\right)$, where $x^{\prime} \cdot y^{\prime}$ is the vector on the unit $n$ sphere obtained by the loop product in $\pi(\mathscr{L})$ of $x^{\prime}$ and $y^{\prime}$. If $x$ or $y$ is the zero vector $O$, define $x \cdot y=O$.

We will now show that $E^{n+1}$, with the operation of multiplication defined in the previous paragraph, the usual Euclidean vector addition, and the usual Euclidean scalar multiplication, is a division algebra over the real numbers. If the usual Euclidean norm is denoted by || , then this norm will satisfy the equation $|x \cdot y|=|x||y|$.

$E^{n+1}$ is a vector space over the reals. We first show that

$$
a(x \cdot y)=(a x) \cdot y=x \cdot(a y)
$$

for any two vectors $x, y$ and any real number $a$. Let $c x^{\prime}$ and $d y^{\prime}$ be the unique 
representations of $x$ and $y$, respectively, described above. If $a$ is positive, then $a(x \cdot y)=a c d\left(x^{\prime} \cdot y^{\prime}\right)=a x \cdot y=x \cdot a y$, since $a c x^{\prime}$ and $a d y^{\prime}$ are the unique representations of $a x$ and $a y$, respectively. If $a$ is zero, all three of the vectors in (2)are the zero vector. If $a$ is negative, we have initially

$$
a x \cdot y=|a|(-x) \cdot y=|a| c d\left(-x^{\prime} \cdot y^{\prime}\right)=|a| c d \pi\left(\pi^{-1}\left(-x^{\prime}\right) \circ \pi^{-1}\left(y^{\prime}\right)\right) .
$$

The purpose of this paragraph is to show that there exists an element $g$ in the center of $\overline{\mathscr{G}}$ such that $g$ has order two and $\pi^{-1}(-x)=g\left(\pi^{-1}(x)\right)$ for all $x$ in $E^{n+1}$. Since $\mathscr{L}$ is a Hopf space, $n=1,3$, or 7 . Montgomery and Samelson [8] and A. Borel [2] have shown that $\overline{\mathfrak{5}}$ must be isomorphic to one of the following groups: for $n=1, \mathrm{SO}(2)$; for $n=3, \mathrm{SU}(2), \mathrm{SO}(4)$, and $\mathrm{Sp}(1)$; for $n=7, \mathrm{SU}(4), \mathrm{SO}(8)$, $\mathrm{Sp}(2)$, and the covering group of $\mathrm{SO}(7)$. It is claimed that each one of the above groups has an element $g$ of order two in its center for which $g(x) \neq x$ for all $x \in S^{n}$. In [3, p. 414] it is seen that there is an element $g$ of order two in the center of each of the above groups (and only one such $g$ ). For some $z \in S^{n}, g(z) \neq z$ because of effectiveness. Furthermore, if for some $y \in S^{n}, g(y)=y$, then there exists $h \in \overline{\mathfrak{G}}$ such that $h(y)=z$ by transitivity. Hence

$$
z \neq g(z)=g(h(y))=h(g(y))=h(y)=z,
$$

establishing the above claim. Furthermore,

$$
H(g)(H(g)(x)+x)=[H(g)](H(g)(x))+H(g)(x)=x+H(g)(x)
$$

for $x \in E^{n+1}$. Every element of $\mathrm{SO}(n+1)$ leaves the origin fixed. If $H(g)(x)+x$ is not the zero vector, then the line through the origin and $H(g)(x)+x$ is left fixed by $H(g)$. There is an element $p \in \pi\left(S^{n}\right)$ which is left fixed since $\pi\left(S^{n}\right)$ is a sphere about the origin. But $H(g)(p)=p$ contradicts the fact that $g(x) \neq x$ for all $x \in S^{n}$. Hence $H(g)(x)=-x$ and $\pi^{-1}(-x)=\pi^{-1}(H(g)(x))=g\left(\pi^{-1}(x)\right)$.

Letting $z=\pi^{-1}\left(y^{\prime}\right)$ the following equations hold:

$$
\begin{aligned}
|a| c d & \pi\left(\pi^{-1}\left(-x^{\prime}\right) \circ \pi^{-1}\left(y^{\prime}\right)\right) \\
& =|c| c d \pi\left(g\left(\pi^{-1}\left(x^{\prime}\right)\right) \circ z\right) \\
& =|a| c d \pi\left(R_{z}\left(g\left(\pi^{-1}\left(x^{\prime}\right)\right)\right)\right)=|a| c d \pi\left(g\left(R_{z}\left(\pi^{-1}\left(x^{\prime}\right)\right)\right)\right) \\
& =|a| c d\left(-\pi\left(\pi^{-1}\left(x^{\prime}\right) \circ z\right)\right)=|a| c d\left(-\left(x^{\prime} \cdot y^{\prime}\right)\right)=\operatorname{acd}\left(x^{\prime} \cdot y^{\prime}\right)=a(x \cdot y)
\end{aligned}
$$

Similarly it can be shown that $x \cdot a y$ also equals $a(x \cdot y)$ by using left translates in $\overline{(5}$. Thus equation (2) holds.

Furthermore, multiplication is left distributive, since

$$
x \cdot(y+z)=a x^{\prime} \cdot(y+z)=a b \pi\left(\pi^{-1}\left(x^{\prime}\right) \circ \pi^{-1}(y / b+z / b),\right.
$$

where $x=a x^{\prime}$ and $y+z=b t$ are the unique representations described above 
for $x$ and $y+z$, respectively. We may assume that $x$ and $y+z$ are not the zero vector. Letting $u=\pi^{-1}\left(x^{\prime}\right)$, it follows further that

$$
\begin{aligned}
a b \pi\left(L_{u}\left(\pi^{-1}\left(\frac{1}{b} y+\frac{1}{b} z\right)\right)\right) \\
\quad=a b\left(\left[H\left(L_{u}\right)\right]\left(\pi\left(\pi^{-1}\left(\frac{1}{b} y+\frac{1}{b} z\right)\right)\right)\right) \\
=a b\left(\left[H\left(L_{v}\right)\right]\left(\frac{1}{b} y\right)+\left[H\left(L_{u}\right)\right]\left(\frac{1}{b} z\right)\right)=a\left[H\left(L_{u}\right)\right](y)+a\left[H\left(L_{u}\right)\right](z) \\
=a \pi\left(L_{u}\left(\pi^{-1}(y)\right)\right)+a \pi\left(L_{u}\left(\pi^{-1}(z)\right)\right)=a\left(x^{\prime} \cdot y\right)+a\left(x^{\prime} \cdot z\right) \\
=\left(a x^{\prime}\right) \cdot y+\left(a x^{\prime}\right) \cdot z=x \cdot y+x \cdot z .
\end{aligned}
$$

In a similar manner, it can be shown that multiplication distributes over vector addition on the right by using right translates.

The equations $u \cdot x=v$ and $y \cdot u=v$ in $R^{n+1}$ may be solved for $x$ and $y$, if $u$ and $v$ are not the zero vector, by letting $x=(a / b)\left(u^{\prime-1} v^{\prime}\right)$ and $y=(a / b)\left(v^{\prime} u^{\prime-1}\right)$, where $u=b u^{\prime}$ and $v=a v^{\prime}$ are the representations described above.

Finally, the norm defined above satisfies the equation $|x||y|=|x \cdot y|$; for $|x \cdot y|=a b=|x||y|$, where $x=a x^{\prime}$ and $y=b y^{\prime}$ are the representations described above.

It is known that such a division algebra as $R^{n+1}$ (with $n>0$ ) is either the complex numbers, the quaternions, or the Cayley numbers [12]. Therefore $\mathscr{L}$, being isomorphic to the subloop of $R^{n+1}$ of norm one, is determined.

4. Certain loops on $R^{n}$. A topological loop with zero is a Hausdorff space $\mathscr{L}$ with a continuous multiplication and an element $O \in \mathscr{L}$ which satisfies the following conditions:

(1) $\mathscr{L} \backslash O$ is a topological loop with respect to the multiplication;

(2) $O x=x O=O$ for all $x \in \mathscr{L}$;

(3) $\mathscr{L} \backslash O$ is dense in $\mathscr{L}$.

The study of locally compact, connected loops with zero was initiated by K. H. Hofmann [7] and the study of locally compact, connected groups with zero (two-ended groups) by H. Freudenthal and L. Zippin [13].

THEOREM 5. Let $R^{n}$ be a loop with zero (assume that the origin $O$ of $R^{n}$ is the zero) and suppose that some topological $(n-1)$-sphere $S^{n-1}$ about the origin containing the identity of $R^{n}$ satisfies $\delta(g x, g y)=\delta(x, y)=\delta(x g, y g)$ for all $g \in S^{n-1}$ and all $x, y$ in $R^{n}$, where $\delta$ is a metric on $R^{n}$ compatible with its usual topology. Then $S^{n-1}$ is a subloop of $R^{n}$ and $S^{n-1}$ is determined by Theorem 4 . 
Proof. Let $H$ denote the subloop of $R^{n}$ generated by $S^{n-1}$. It will be shown that the closure of $H, \bar{H}$, is a compact subloop of $R^{n}$ and is also connected if $n>1$.

Since $\bar{H}$ is closed in $R^{n}$, it is sufficient to show that if $\left\{g_{i}\right\}$ is a sequence in $H$, then $\left\{g_{i}\right\}$ does not converge to $\infty$ or 0 , where $\infty$ is the "ideal point" in the onepoint compactification of $R^{n}$. Suppose $\lim _{i} g_{i}=O$. We have

$$
g_{i}=R_{x_{1}} R_{x_{2}}^{-1} L_{x_{3}} L_{x_{4}}^{-1} \cdots L_{x_{s-1}} L_{x_{s}}^{-1}(1)=f_{i}(1) \text { for some } x_{j} \in S^{n-1}, j \leqq s .
$$

Then $\delta(1,0)=\delta\left(f_{i}(1), f_{i}(0)\right)=\delta\left(g_{i}, 0\right)$ for all $i$ and consequently $\delta(1,0)=0$; but this equality cannot hold. Also suppose that $\lim _{i} g_{i}=\infty$. By [7, Proposition 3.12] $\lim _{i}\left(g_{i}^{-1} 1\right)=0$, again leading to a contradiction. Furthermore a modification of the proof of Proposition 2.12 of [7] implies that $H$ is arcwise connected if $n>1$, Since $H$ is generated by an arcwise connected set containing 1 . Consequently $\bar{H}$ is a compact, connected (for $n>1$ ) loop containing $S^{n-1}$ and $H$.

If $n=1$, let $1 \neq g \in S^{n}$. If $I$ is the closed interval $[g, 1]$, then $g I$ is an interval with endpoints $g$ and $g^{2}$ and with $O$ as an inner-point; hence $g I=\left[g, g^{2}\right]$ with $g^{2}>O$. We use the usual order on the real numbers. The component $R^{+}$of 1 in $R^{1} \backslash O$ is a subloop containing $g^{2}=h$. The following argument of Hofmann (unpublished) shows that if $h>1$, then $1<h<h^{2}<\left(h^{2}\right)^{2}<\cdots$. In $R^{+} \times R^{+}$ let $A$ be the graph of the relation $<$ and let $\Delta$ be the diagonal. For $(x, y) \in R^{+} \times R^{+}$define $(x, y) h=(x h, y h)$. Then $h$ is a homeomorphism of $R^{+} \times R^{+}$ and either $A h \subset A$ or $A h \subset R^{+} \times R^{+} \backslash(A \cup \Delta)$. If $h>1$, then $\left(1 h^{-1}\right)$ must be less than 1 . Then $\left(\left(1 h^{-1}\right), 1\right) h=(1, h)$. We have, therefore, that $h$ maps one element of $A$ into $A$. Hence $A h \subset A$. The above inequalities then hold as claimed. $H$ being compact implies that the sequence $\left\{1, h, h^{2},\left(h^{2}\right)^{2}, \cdots\right\}$ converges to an idempotent which must be 1 . Hence $h$ cannot be greater than 1 . Similarly $h \nless 1$. Then $h=g^{2}=1$ and $S^{0}$ is a subloop.

Now assume $n>1$. Let $\overline{\mathfrak{5}}$ be the group of all translates of $\bar{H}$. Then $\overline{\mathfrak{F}}$ is a compact, connected group of homeomorphisms acting effectively and transitively on $\bar{H}$. It is easily seen that $\bar{H}$ is $(n-1)$-dimensional and topologically equal to $\overline{\mathfrak{G}} / \overline{\mathfrak{F}}_{1}$. It follows that $\bar{H}$ is locally the topological product of an $(n-1)$-cell and a totally disconnected set [9,p. 239]. Let $\mathscr{U}$ denote the relative topology in $R^{n}$ of $\bar{H}$ and $(\bar{H}, \mathfrak{M}(\mathscr{U}))$ denote the space obtained from $(\bar{H}, \mathscr{U})$ with the associated locally arcwise connected topology $[5]$. Then $(\bar{H}, \mathfrak{M}(\mathscr{U}))$ is an locally Euclidean space. If $C$ denotes the component of 1 in $(\bar{H}, \mathfrak{M}(\mathscr{U})), C$ is an $(n-1)$-dimensional manifold. Also $C$ contains $S^{n-1}$ and $H$ [5, p. 634, §3.2]. It follows that $C=H=S^{n-1}$. Since $S^{n-1}$ has an invariant metric, Theorem 4 may now be applied.

A loop is called diassociative if every pair of elements belongs to a subgroup.

THEOREM 6. Let $R^{n}$ be as in Theorem 5. Furthermore let $R^{n} \backslash O$ be diassociative. Then $R^{n} \backslash O$ is the direct product of a subgroup $M$ isomorphic to the positive real numbers under multiplication and $S^{n-1}$, which is isomorphic to 
either the cyclic group of order two, or the complex, the quaternion, or the Cayley numbers of norm one, respectively.

Proof. K. H. Hofmann [7] has shown the following:

(a) $R^{n} \backslash O$ has a closed subgroup isomorphic to the positive real numbers with the usual multiplication;

(b) if $M$ denotes any subgroup of the type in (a), then $R^{n} \backslash O=M S$, where $S=\left\{x \in R^{n} \mid O: \Gamma(x)\right.$ is compact in $\left.R^{n} \backslash O\right\}$, and $\Gamma(x)$ is the closed subgroup generated by $x$;

(c) $R^{n} \backslash O$ is homeomorphic to $M \times S$, and $S$ is compact in $R^{n} \backslash O$.

We have that $R^{n} \mid O$ is homeomorphic to both $M \times S^{n-1}$ and $M \times S$, implying that $\operatorname{dim} S=\operatorname{dim} S^{n-1}$. Theorem 5 concludes that $S^{n-1}$ is a subloop, and we also know that $S^{n-} \subset S$. Thus $S^{n-1}$ is homogeneous and open in $S$. Connectedness of $S$ implies $S^{n-1}=S$.

We first establish that for a subgroup $M$ as described above

$$
\left(m_{1} m_{2}\right) s=m_{1}\left(m_{2} s\right)
$$

for $m_{1}, m_{2} \in M$ and $s \in S$. Let $p$ and $q$ be integers. Then $m_{1}\left(m_{1}{ }^{p / q} s\right)=\left(m_{1} m_{1}{ }^{p / q}\right) s$ since $m_{1}^{1 / q}, m_{1}^{p / q}, m_{1}$, and $s$ all belong to the subgroup generated by $m_{1}^{1 / q}$ and $s$. Since $\left\{m_{1}^{p / q}: p\right.$ and $q$ are integers $\}$ is dense in $M$, (3) follows.

Also for such a subgroup as $M$, we will show that each element of $R^{n} \backslash O$ can be expressed uniquely as the product of an element from $M$ and an element from $S$. Suppose $g=m s=n t$ for $m, n \in M$ and $s, t \in S$. Then $m^{-1}(n t)=s=\left(m^{-1} n\right) t$ and $s t^{-1}=m^{-1} n$. Since $M \cap S=\{1\}$, it follows that $m=n, s=t$, and $g$ is represented uniquely.

The existence of a subgroup $M$ as in (a) with the property that $m s=s m$ for $m \in M$ and $s \in S$ is next established. Let $N$ be a subgroup as in (a). The following two notations are used: for $s \in S, O(s)$ is the order of $s$, and $\langle A, B\rangle$ is the closure in $R^{n}$ of the subloop of $R^{n} \backslash O$ generated by the sets $A$ and $B$. We use also the following properties of the Cayley numbers of norm 1 : if $C_{1}, C_{2}$, and $C_{3}$ are three different circle subgroups, $\left\langle C_{1}, C_{2}\right\rangle$ is the 3-sphere group; and if $C_{3} \notin\left\langle C_{1}, C_{2}\right\rangle$, then $\left\langle C_{1}, C_{2} \cup C_{3}\right\rangle$ is the Cayley numbers of norm 1 . Let $C$ be a circle group contained in $S$. Then $\langle C, N\rangle$ is a connected, locally compact loop with zero. Using methods similar to the verification of (3), $\langle C, N\rangle$ is also a group with zero. According to the theory of such groups, $\langle C, N\rangle \mid O$ is the direct product of $P$ and $K, P$ isomorphic to the positive real numbers, and $K$ a compact, connected group. If for each such circle group in $S,\langle C, N\rangle$ is two-dimensional, then it follows that $N$ commutes with $S$, and $M$ is defined to be $N$.

However, if there exists a circle group $C$ such that $\langle C, N\rangle$ is not twodimensional, then $\langle C, N\rangle \mid O$ is the direct product of $P$ and $K$ as above and is four-dimensional since $K$ must be three-dimensional. Also this compact direct factor $K$ is isomorphic to the quaternions of norm one. Define $P=M$. It is now 
shown that $M$ commutes elementwise not only with elements of $K$ but with all elements of $S$. If $C_{1}$ is a circle group not contained in $K$, let $\left\langle C_{1}, M\right\rangle \mid O$ be expressed as the direct product of $L$ and $T, L$ isomorphic to the positive real numbers and $T$ compact and connected. If $T$ is one-dimensional, $M$ commutes with $T$ element for element, and it follows that $M$ commutes in the same manner with $\langle T, K\rangle=S$. We may thus suppose that $T$ is three-dimensional and a three-sphere group. Every subgroup of the quaternions which is isomorphic to the positive real numbers commutes with some circle group, so there is a circle group $C_{2}$ which commutes element for element with $M$. If $C_{2}$ is not contained in $K$, then again $M$ commutes with all of $S$. We may finally suppose that $C_{2} \subset K \cap T$. This supposition will be contradicted. Let $C^{\prime}$ be a circle group in $T$ different from $C_{1}$ and let $C^{\prime \prime}$ be a circle group in $K$ different from $C_{2}$. Also let $j \in L, m \in M, c_{1} \in C^{\prime}$, $d_{1} \in C^{\prime \prime}$ with the properties that $m \neq 1 \neq j$ and that $1<o\left(c_{1}\right)=o\left(d_{1}\right)<\infty$. Let $G_{1}$ be the subgroup generated by $j c_{1}$ and $m d_{1}$. For each integer $n>1$, let $G_{n}$ be the subgroup generated by $j^{\alpha_{n}} c_{n}$ and $m^{\alpha_{n}} d_{n}$, where the following are satisfied: $c_{n} \in C^{\prime}$ and $c_{n}^{2}=c_{n-1}, d_{n} \in C^{\prime \prime}$ and $d_{n}^{2}=d_{n-1}, \quad\left\{c_{n}\right\}$ and $\left\{d_{n}\right\}$ converge to 1 , $\alpha_{n}=\left(2^{n-1}(1+2 q)(1+4 q) \cdots\left(1+2^{n-1} q\right)\right)^{-1}$, and finally $q=o\left(c_{1}\right)$. Then it follows that $G_{n} \supset G_{n-1}$. Let $H$ be the closure of $\bigcup\left\{G_{n}: n=1,2, \cdots\right\}$. For each $n$

$$
\begin{aligned}
\left(m^{\alpha_{n}} c_{n}\right)^{2^{n-1} q} & =m^{q /\left((1+2 q) \ldots\left(1+2^{n-1} q\right)\right)} c_{n}{ }^{2^{n-1} q} \\
& =m^{q /\left((1+2 q) \ldots\left(1+2^{n-1} q\right)\right)}
\end{aligned}
$$

and this element belongs to $H$. Because elements of $M$ arbitrarily close to 1 belong to $H, M \subset H$. Hence $C^{\prime} \subset H$. Similarly, $L$ and $C^{\prime \prime}$ are contained in $H$. But the product of $M$ and $L$ contains $C_{2}$, as the following argument shows. Recall that we have assumed that $M \cup L \cup C_{2}$ is contained in the product of $L$ and $T$ and that $M$ commutes with $C_{2}$. Since $M \neq L, 4 \geqq \operatorname{dim}\langle M, L\rangle \geqq 2$, and there is a circle subgroup $C_{3}$ of $\langle M, L\rangle$. If $\operatorname{dim}\langle M, L\rangle=2$, elements of $M$ commute with elements of $C_{3}$. Either $\left\langle C_{2}, C_{3}\right\rangle=T$ and $M=L$ (this is not so), or else $\left\langle C_{2}, C_{3}\right\rangle=C_{3}$ and $C_{2} \subset\langle M, L\rangle$. The dimensiom of $\langle M, L\rangle$ cannot be 3 . If $\operatorname{dim}\langle M, L\rangle=4$, $C_{2} \subset T=\langle M, L\rangle$.

Now we have that $C_{2} \cup C^{\prime} \cup C^{\prime \prime} \subset H$. But $\left\langle C_{2}, C^{\prime}\right\rangle=T$ and $\left\langle C_{2}, C^{\prime \prime}\right\rangle=K$. $T$ and $K$, being different maximal subgroups of $S$, cannot generate a subgroup of $S$. The supposition that $C_{2} \subset K \cap T$ is contradicted, and $M$ has the desired properties.

It is now shown that for $m \in M$ and $s_{1}, s_{2} \in S$

$$
\left(m s_{1}\right) s_{2}=m\left(s_{1} s_{2}\right) \text {. }
$$

There exist in $S$ elements $t_{i}$ for each natural number $i$, of finite order $p_{i}$, such that $\left\{t_{i}\right\}$ converges to $s_{2}$ and $\left\{p_{i}\right\}$ converges to $\infty$. Both $m^{1 / p_{i}} t_{i}$ and $s_{1}$ belong to a group. Because of the commutative law, $\left(m^{1 / p_{i}} t_{i}\right)^{p_{i}}=m$. Hence $m, m^{1 / p_{i}} t_{i}$, and $s_{1}$ associate. By continuity of multiplication (4) follows. 
The following equations can be established in a manner similar to the proof of (4):

$$
\begin{aligned}
\left(s_{1} m\right) s_{2} & =s_{1}\left(m s_{2}\right), & & \text { for } m \in M \text { and } s_{1}, s_{2} \in S, \\
m\left(s_{1}\left(n s_{2}\right)\right) & =\left(m s_{1}\right)\left(n s_{2}\right), & & \text { for } m, n \in M \text { and } s_{1}, s_{2} \in S .
\end{aligned}
$$

By equations (3)-(6) and commutativity it follows that $\left(m s_{1}\right)\left(n s_{2}\right)=(m n)\left(s_{1} s_{2}\right)$ for $m, n \in M$ and $s_{1}, s_{2} \in S$, and the proof is concluded.

5. Example. For complex numbers $e^{i \alpha}$ and $e^{i \beta}$ on the unit circle $S^{1}$ in the complex plane, define a binary operation in the following manner:

$$
e^{i \alpha} \circ e^{i \beta}=e^{i(\alpha+\beta+\sin \alpha+\sin \beta)} \text {. }
$$

Then $\left(S^{1}, \circ\right)$ is a commutative loop in which the binary operations are real analytic and which does not have an invariant uniformity. This example may be generalized to $S^{3}$ and $S^{7}$. Hence the hypotheses concerned with invariant uniformities in several of the theorems of this paper are indispensable for the conclusions desired.

\section{REFERENCES}

1. Richard Arens, Topologies for homeomorphism groups, Amer. J. Math. 68 (1946), 593-610.

2. A. Borel, Le plan projectif des octaves et les sphères comme espaces homogènes, C. R. Acad. Sci. Paris 230 (1950), 1378-1380.

3. - Topology of Lie groups, Bull. Amer. Math. Soc. 61 (1955), 397-432.

4. Robert Ellis, Locally compact transformation groups, Duke Math. J. 24 (1957), 119-126.

5. A. Gleason and R. Palais, On a class of transformation groups, Amer. J. Math. 79 (1957), 631-648.

6. K. H. Hofmann, Topologische loops, Topologische loops mit schwachen assoziativitätsforderungen, and Topologische doppelloops, Math. Z. 70 (1958), 13-37, 125-155, 213-230.

7. - Tulane lecture notes, Mimeographed notes printed at Tulane University, 1961.

8. D. Montgomery and H. Samelson, Transformation groups of spheres, Ann. of Math. (2) 44 (1943), 454-470.

9. D. Montgomery and L. Zippin, Topological transformation groups, Interscience, New York, 1955.

10. P. S. Mostert, On a compact Lie group acting on a manifold, Ann. of Math. (2) 65 (1957), 447-455.

11. Jean Poncet, Groupes de Lie compacts de transformations, C. R. Acad. Sci. Paris 245 (1957), 13-15.

12. K. Urbanik and F. B. Wright, Absolute-valued algebras, Proc. Amer. Math. Soc. 11 (1960), 861-866.

13. L. Zippin, Two-ended topological groups, Proc. Amer. Math. Soc. 1 (1950), 309-315.

TULANE UNIVERSITY, New OrLeans, Louisiana 